\title{
Control Strategy for Small Molecule Impurities in Antibody-Drug Conjugates
}

\author{
Hai H. Gong, ${ }^{1}$ Nathan Ihle, ${ }^{2}$ Michael T. Jones, ${ }^{3,6}$ Kathleen Kelly, ${ }^{4}$ Laila Kott, ${ }^{5}$ Thomas Raglione, ${ }^{4}$ \\ Scott Whitlock, ${ }^{2}$ Qunying Zhang, ${ }^{1}$ and Jie Zheng ${ }^{1}$
}

Received 15 December 2017; accepted 16 December 2017; published online 4 January 2018

Abstract. Antibody-drug conjugates (ADCs) are an emerging class of
biopharmaceuticals. As such, there are no specific guidelines addressing impurity limits and
qualification requirements. The current ICH guidelines on impurities, Q3A (Impurities in
New Drug Substances), Q3B (Impurities in New Drug Products), and Q6B (Specifications:
Test Procedures and Acceptance Criteria for Biotechnological/Biological Products) do not
adequately address how to assess small molecule impurities in ADCs. The International
Consortium for Innovation and Quality in Pharmaceutical Development (IQ) formed an
impurities working group (IWG) to discuss this issue. This white paper presents a strategy for
evaluating the impact of small molecule impurities in ADCs. This strategy suggests a science-
based approach that can be applied to the design of control systems for ADC therapeutics.
The key principles that form the basis for this strategy include the significant difference in
molecular weights between small molecule impurities and the ADC, the conjugation
potential of the small molecule impurities, and the typical dosing concentrations and dosing
schedule. The result is that exposure to small impurities in ADCs is so low as to often pose
little or no significant safety risk.

KEY WORDS: ADC; control strategy; small molecule impurity; risk assessment.

\section{INTRODUCTION}

The material in this manuscript was developed with the support of the International Consortium for Innovation and Quality in Pharmaceutical Development (IQ Consortium or IQ). The IQ is a not-for-profit organization composed of pharmaceutical and biotechnology companies with a mission of advancing science-based and scientifically driven standards and regulations for pharmaceutical and biotechnology products, worldwide. Today, IQ represents 42 pharmaceutical and biotechnology companies. Please visit www.iqconsortium.org for more information.

Antibody-drug conjugates (ADCs) are an emerging class of biopharmaceuticals that have demonstrated significant potential to improve clinical outcomes. Because these novel

\footnotetext{
${ }^{1}$ AbbVie Inc., 1 North Waukegan Rd, North Chicago, Illinois 60064-
1802, USA.

${ }^{1}$ AbbVie Inc., 1 North Waukegan Rd, North Chicago, Illinois 60064-
1802, USA.

${ }^{2}$ Seattle Genetics, Inc., 21823 30th Dr SE Bothell, Washington, 98021, USA.

${ }^{3}$ Pfizer, Inc., 700 Chesterfield Parkway West, Chesterfield, Missouri 63017, USA.

${ }^{4}$ Bristol-Myers Squibb, 206 Providence Line Rd, Princeton, New Jersey 08540, USA.

${ }^{5}$ Takeda Pharmaceuticals International Co., 40 Landsdowne St, Cambridge, Massachusetts 02139, USA.

${ }^{6}$ To whom correspondence should be addressed. (e-mail: michael.t.jones@pfizer.com)
}

agents combine both protein and small molecule structural elements into a single therapeutic, there are no established guidelines delineating expectations for their control. An ADC typically consists of a low molecular weight linker covalently attached to both a small molecule drug ${ }^{1}$ and a monoclonal antibody (mAb) (1). The act of attachment of the drug to the antibody is called conjugation. There has been a proliferation of ADC reviews over the last few years, including new peer reviewed journals and books devoted to this topic (2-4). Figure 1 is a cartoon depicting some of the most commonly encountered conjugation technologies. Most ADCs currently in the clinic and all those that have been approved are intended for oncology indications, thus this white paper is focused on considerations for this therapeutic area only. The principles outlined should hold regardless of the ADC conjugation chemistry, but we have used one of the more common ADC technologies to exemplify a sound control strategy. In this approach (Fig. 2), the drug and linker are combined by chemical synthesis to form a linker-drug

\footnotetext{
1 In this manuscript, the term "drug" is used to describe the pharmacologically active small molecule that is conjugated to a monoclonal antibody via a linker. Other names for this species have included payload, cytotoxin, toxin, and warhead. The term "linker-drug" refers to a single molecular entity that contains both the linker and the drug. Other names that have been used for this species include drug-linke and linker/payload.
} 


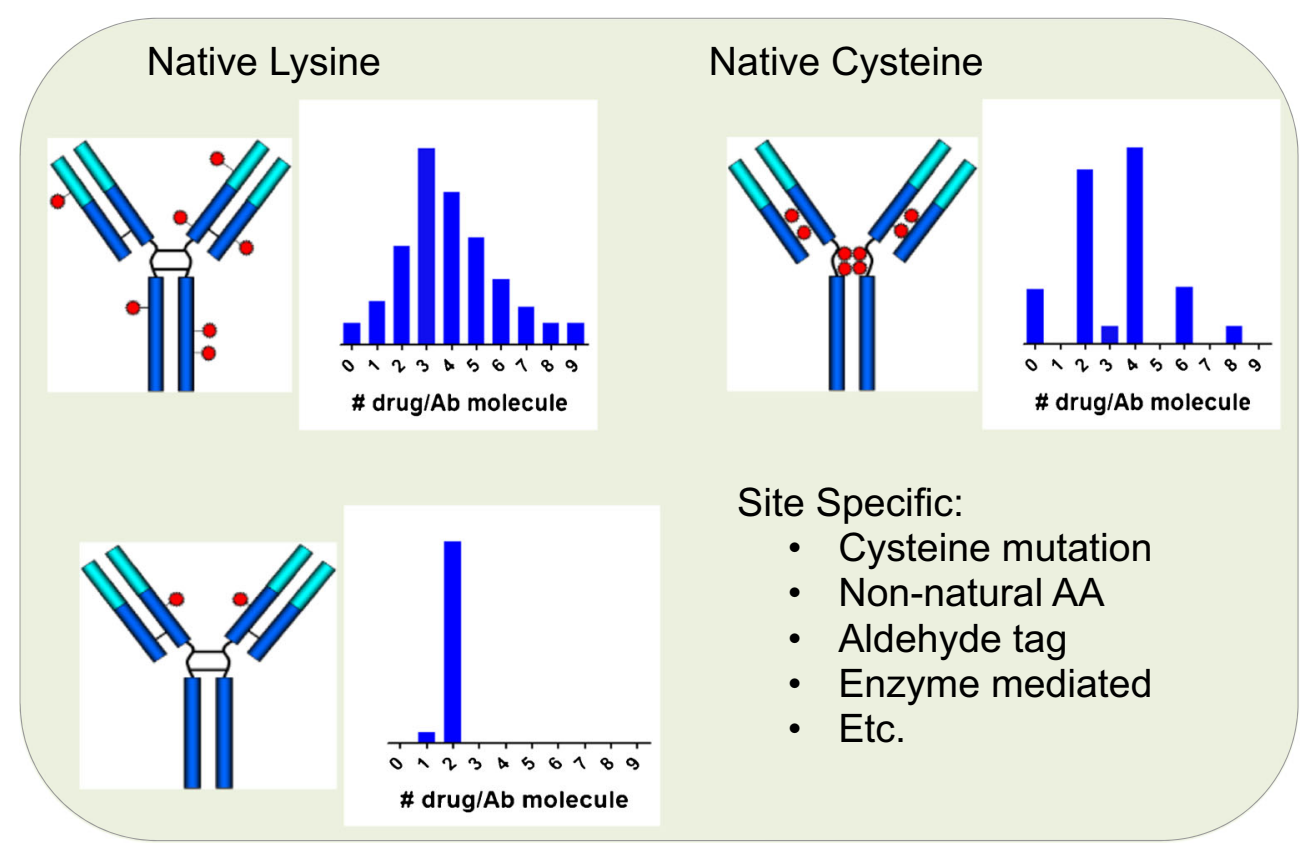

Fig. 1. A cartoon representation of different conjugation chemistries for ADCs. The linker is attached between the drug and the mAb usually by a covalent bond through a cysteine or lysine

intermediate which is then conjugated directly to a mAb. Other approaches, including a two-step process in which a linker is first attached to the mAb followed by a separate step to attach the drug, can be evaluated following similar logic but will not be explicitly addressed in this paper. The chemical reactions involved in the conjugation process usually occur in biological buffer solutions; however, organic solvent may be added to boost the solubility of the linker-drug. The crude ADC then undergoes a purification and formulation step (typically ultrafiltration/diafiltration or UF/DF) for removal of small molecule impurities including solvents, reagents, elemental impurities, and non-conjugated linkerdrug-related components. These general manufacturing processes provide the background for the discussion of small molecule impurities.

Like other drug substances (DS) and drug products (DP), impurities can arise during the synthesis, purification, and storage of the intermediates as well as the ADC DS and DP. Depending on their point of origin and molecular weight, impurities in ADCs can be classified as mAb-related or small molecule. The mAb-related impurities will not be addressed in this paper. Information provided in $\mathrm{ICH}_{\mathrm{Q}} \mathrm{QB}^{2}$ details impurity and heterogeneity concerns for $\mathrm{mAbs}$, and these will typically need to be addressed during the manufacture of the $\mathrm{mAb}$ intermediate. Some of these attributes, such as aggregates and charge variants, are also relevant to the DS and/or DP but are beyond the scope of this paper. Here we will concentrate on the impurities that are associated with the small molecule component of the ADC. These are impurities that originate during the manufacture of the linker, the drug, or the linker-drug, and the related small molecule impurities that arise during

2 ICH Q6B: Specifications: Test Procedures and Acceptance Criteria for Biotechnological/Biological Products the manufacture or storage of the ADC DS and DP. These small molecule impurities typically have molecular weights of $<2000 \mathrm{Da}$ (the molecular weight of a typical linker-drug) while the ADC itself will have a molecular weight of $\sim 160,000 \mathrm{Da}$ (typical for ADCs with a drug-toantibody ratio (DAR) of 4). This difference in molecular weight means that any small molecule impurity present in the ADC, including those originating in the linker-drug intermediate, represents a much smaller proportion of the total administered dose of ADC than would be the case in a typical small molecule pharmaceutical. This difference should play a significant role in guiding decisions about risks associated with these impurities and the appropriate controls. Other factors that play a part in the assessment include the dose, schedule, duration of therapy, and therapeutic area in which the ADC is being applied. All these factors will be discussed as we propose an integrated approach to the development of a control strategy for small molecule impurities in ADCs.

\section{Current Regulatory Guidance}

For a typical small molecule new chemical entity (NCE), ICH $\mathrm{Q}^{3} \mathrm{~A}^{3}$ and $\mathrm{Q}^{3} \mathrm{~B}^{4}$ provide guidelines for control of impurities. In particular, they clearly define the acceptable levels of organic (drug related) impurities and actions that are required in order to justify their presence at higher levels in a DS (Table 1) or DP (Table 2). Biological/biotechnological products are explicitly exempted from these guidances; however, we propose that the principles outlined, especially with respect to acceptable levels of small molecule impurities, are appropriate to use to guide the assessment of small

3 ICH Q3A: Impurities in New Drug Substances

${ }^{4} \mathrm{ICH}$ Q3B: Impurities in New Drug Products 


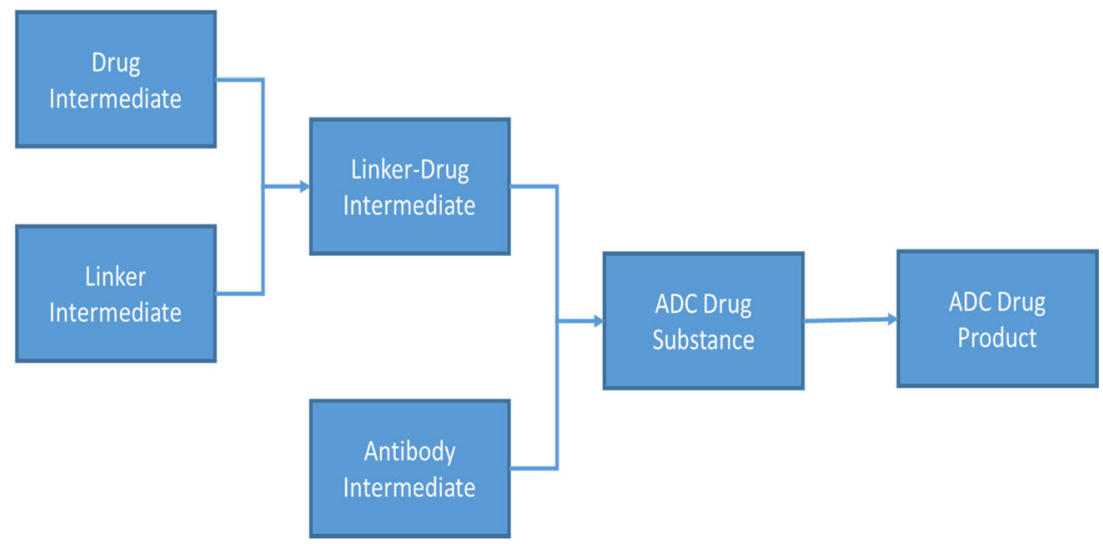

Fig. 2. Convergent manufacturing scheme for ADCs

molecule impurities in ADCs. It is worth noting that many drugs used in forming ADCs belong to a class of compounds called cytotoxins. Cytotoxins can damage or kill healthy cells leading to unacceptable toxicity, so their presence as impurities may be of special concern. In order to define an appropriate control strategy, it is important to understand the level of risk these components would pose if they were to be present as impurities in an ADC, and what levels might be of minimal concern. The guidance points out that the threshold for considering an impurity "unusually potent" is if it would be expected to produce a toxic or pharmacological effect at a level not more than the identification threshold $(0.10 \%$ in $\mathrm{ICH}$ Q3A(R2)). This concern must be addressed, but in the context of the particular clinical setting in which ADCs are to be utilized, and this will be discussed in later sections. Most of the small molecule impurities, including the free drug, would not fall in the category of being unusually potent. For example, if the ADC is dosed at $2 \mathrm{mg} / \mathrm{kg}$ and the identification threshold is $0.1 \% \mathrm{wt} / \mathrm{wt}$ than any impurity that was not pharmacologically active or toxic at $2 \mu \mathrm{g} / \mathrm{kg}$ would not be "unusually potent."

ICH Q3C ${ }^{5}$ details expectations for control of residual solvents. ICH $\mathrm{Q}^{2} \mathrm{D}^{6}$ provides guidance with respect to elemental impurities. These guidelines should be considered during the development of ADCs.

$\mathrm{ICH} \mathrm{M}^{7}$ provides guidance on the assessment and control of genotoxic impurities. However, this guidance details how adherence to the suggested limits may not be appropriate in the case of treating severe, life-threatening diseases or where the duration of treatment is short (Table 3).

ICH S9 ${ }^{8}$ explains how controlling impurities to levels that provide negligible risk is not an appropriate requirement for pharmaceuticals used to treat advanced cancer patients. For these situations, the risk-benefit assessment is different from that applied

\footnotetext{
5 ICH Q3C: Impurities: Guidance for Residual Solvents

${ }^{6}$ ICH Q3D: Guidelines for Elemental Impurities

7 ICH M7: Genotoxic Impurities: Assessment and Control of DNA Reactive (Mutagenic) Impurities in Pharmaceuticals to limit Potential Carcinogenic Risk

8 ICH S9: Nonclinical Evaluation for Anticancer Pharmaceuticals
}

to drugs used in other indications. Since most ADCs are currently being developed for use in advanced cancer indications, ICH S9 should be applicable. Therefore, for most ADCs, deviation from the limits established in ICH Q3(A, B, C and D) and ICH M7 can be justified.

In cases where ADCs are to be developed for diseases that are not covered by ICH S9, adjustments to the strategies proposed below may be considered. The current ICH S9 Q\&A draft document ${ }^{9}$ addresses these issues and interested readers are referred to that document for further details.

\section{Assumptions in Establishing Control Limits for Small Molecule Impurities in ADCs}

\section{CONTROL OF SMALL MOLECULE IMPURITIES IN ADCS}

In order to simplify the discussion here, we will adopt a number of assumptions:

- $\quad$ The ADCs are intended for use in treating cancer.

- The small molecule drug would be toxic to patients if present at high enough doses.

- The worst-case assumption, that all small molecule impurities containing structural elements of the drug will have a similar level of toxicity as the drug itself, will be used.

- Data is available to demonstrate that the small molecule drug does not need to be considered unusually potent as defined in ICH Q3A (the drug is not pharmacologically active or toxic at $1 \mathrm{mg} / \mathrm{day}$ patient exposure).

\section{Impurity Control in the Linker-Drug Intermediate}

In most cases, the majority of the small molecule impurities present in the DS and DP originate from the linker-drug intermediate, and this is true regardless of whether the impurities are conjugated to the $\mathrm{mAb}$ or not. In keeping with the modern pharmaceutical development principles, impurity control is

\footnotetext{
9 S9 Implementation Working Group ICH S9 Guideline: Nonclinical Evaluation for Anticancer Pharmaceuticals Questions and Answers
} 
Table 1. ICH Q3A(R2) Threshold Levels for Impurities. Qualification Threshold Is the Limit Above Which an Unknown Impurity Will Need to be Qualified

\begin{tabular}{llll}
\hline Dose & Reporting threshold $(\%)$ & Identification threshold & Qualification threshold \\
\hline$\leq 2 \mathrm{~g} /$ day & 0.05 & $0.1 \%$ or $1 \mathrm{mg} /$ day (whichever is lower) & $0.15 \%$ or $1.0 \mathrm{mg} /$ day (whichever is lower) \\
$>2 \mathrm{~g} /$ day & 0.03 & $0.05 \%$ & 0.05 \\
\hline
\end{tabular}

generally best achieved near the point of introduction or at a step where the impurity can be removed. Thus, small molecule impurities in ADCs are generally best controlled during the manufacture of the linker-drug intermediate. These impurities include residual solvents, reagents and their by-products, elemental impurities, and impurities structurally related to the linker, drug, or linker-drug. Most of these impurities are not capable of conjugating to the antibody intermediate, so any residual impurity that is present in the linker-drug intermediate will normally be removed during the UF/DF purification steps commonly utilized during DS manufacture. In this way, meeting the residual solvent and elemental impurities limits in DS and DP, as defined by $\mathrm{ICH}$ Q3C and Q3D respectively, is relatively straight forward (5). ${ }^{10}$ Due to the efficiency of the typical UF/DF purification step, non-conjugatable impurities are expected to be present at levels below those associated with potential risk to patients. Therefore, limits on the levels of these impurities in the linker-drug intermediate should be based solely on a demonstration of process control. In practice, this means that the linker-drug intermediate may not need to meet the solvent and elemental impurity limit guidelines, and more flexibility may be appropriate in demonstrating overall control in the DS and DP. In this way, it should be possible to minimize the inclusion of tests for residual solvent, elemental impurities, and other non-conjugating impurities in the specification for linker-drug intermediates.

The impurities in the linker-drug that have the potential to conjugate to the mAb cannot be removed after conjugation because UF/DF, and other purification steps would typically not provide for separation from the other proteinaceous materials. Therefore, the final opportunity to control these impurities is at the stage of the linker-drug manufacture and release. Understanding how the quantity of these impurities in the intermediate relates to their levels in DS and DP is necessary for the determination of a sound control strategy.

The linker and drug by themselves make up a small fraction of the total mass of the ADC. The average molecular weight of linker and of the drug is approximately $1000 \mathrm{Da}$ each, and we can

\footnotetext{
10 The concentration of small molecule impurities $\left(\mathrm{C}_{\mathrm{sm}}\right)$ decreases during diafiltration. The amount of small molecules purged from the system through a UF/DF step can be calculated based on the following equation: ?

$$
C_{\mathrm{sm}}=C_{\mathrm{sm} 0} \mathrm{e}^{-\mathrm{N}}(1-R)
$$
}

$?$

where $C_{\mathrm{sm} 0}$ is the initial contaminant concentration and $\mathrm{N}$ is the number of diafiltration volumes. For a contaminate with a retention of 0 (which should be true for small molecules well below the membrane cutoff), 6.9 diafiltration volumes will purge $99.9 \%$ of these impurities from the DS use the assumption that most impurities present in these components will have approximately the same molecular weight. A typical ADC, with a DAR of 4 , will have an approximate molecular weight of $160,000 \mathrm{Da}$, and in this case, $<5 \%$ of the mass would derive from the small molecule components with the remaining $95 \%$ originating from the protein. Due to this difference in molecular weights, impurities in the small molecules, if carried through the entire process so that they end up in the DS and DP, will constitute an extremely small quantity of the administered dose. In effect, the conjugation step amounts to a 1:20 dilution of the linker-drug, as well as for impurities (including those that can conjugate to the mAb). If we apply the worst-case scenario that all of the drug-related impurities in the linker-drug intermediate are carried through the process to the drug product, Eq. 1 can be used to calculate the patient's exposure as a function of the impurity level in the linker-drug intermediate prior to the conjugation reaction. The impurity dose is equivalent to the ADC dose times the amount of the impurity in the linker-drug intermediate, times the DAR, and then multiplied by the ratio of the molecular weight of the linkerdrug impurity relative to the molecular weight of the ADC. Dividing by the dose frequency allows one to calculate what the daily impurity exposure would be.

$$
\begin{aligned}
& \text { Daily Impurity Dose }\left(\frac{\mathrm{mg}}{\text { day }}\right) \\
& =\frac{\text { Dose }(\mathrm{mg}) \times \frac{\text { Impurity } \%}{100} \times \text { DAR } \times \frac{\text { Impurity MW }}{\text { ADC MW }}}{\text { Dose Frequency (days) }}
\end{aligned}
$$

Table 2. ICH Q3B(R2) Thresholds for Degradation Products in New Drug Products

\begin{tabular}{ll}
\hline Maximum daily dose & Threshold \\
\hline $\begin{array}{l}\text { Reporting thresholds } \\
\quad \leq 1 \mathrm{~g}\end{array}$ & $0.1 \%$ \\
$\quad>1 \mathrm{~g}$ & $0.05 \%$ \\
Identification thresholds & \\
$\quad<1 \mathrm{mg}$ & $1.0 \%$ or $5 \mu \mathrm{g}$ TDI, whichever is lower \\
$\quad 1-10 \mathrm{mg}$ & $0.5 \%$ or $20 \mu \mathrm{g}$ TDI, whichever is lower \\
$\quad>10-2 \mathrm{~g}$ & $0.2 \%$ or $2 \mathrm{mg}$ TDI, whichever is lower \\
$\quad>2 \mathrm{~g}$ & $0.10 \%$ \\
Qualification thresholds & \\
$\quad<10 \mathrm{mg}$ & $1.0 \%$ or $50 \mu \mathrm{g}$ TDI, whichever is lower \\
$\quad 10-100 \mathrm{mg}$ & $0.5 \%$ or $200 \mu \mathrm{g}$ TDI, whichever is lower \\
$\quad>100 \mathrm{mg}-2 \mathrm{~g}$ & $0.2 \%$ or $3 \mathrm{mg}$ TDI, whichever is lower \\
$>2 \mathrm{~g}$ & $0.15 \%$ \\
\hline
\end{tabular}


Table 3. Acceptable Intakes for Genotoxic Compounds from ICH M7

\begin{tabular}{ll}
\hline Duration of treatment & Daily intake \\
\hline$\leq 1$ month & $120 \mu \mathrm{g} / \mathrm{day}$ \\
$>1-12$ months & $20 \mu \mathrm{g} / \mathrm{day}$ \\
$>1-10$ years & $10 \mu \mathrm{g} / \mathrm{day}$ \\
$>10$ years & $1.5 \mu \mathrm{g} / \mathrm{day}$ \\
\hline
\end{tabular}

Table 4 illustrates how impurity doses would vary as a function of their level in the linker-drug intermediate. In this example, a small molecule impurity weighing 2000 Da present at the level of $3 \mathrm{wt} \%$ in the drug-linker intermediate would result in a $0.15-w t \%$ impurity in the ADC. If the ADCs were dosed at a level of $50 \mathrm{mg}$ once every 3 weeks, the resulting impurity dose would be $75 \mu \mathrm{g}$, and the average daily dose for the impurity would be $3.6 \mu \mathrm{g}$. This is well below the $200 \mu \mathrm{g}$ total daily intake (TDI) that ICH Q3B indicates would require qualification of the impurity (Table 2). On the other hand, $0.15 \%$ is right at the qualification level defined in $\mathrm{ICH}$ Q3A. Thus, any impurity that was observed at levels of 3\% in the linker-drug intermediate would require qualification. For most ADCs under development, manufacturing processes are capable of delivering linker-drug intermediates with individual impurities controlled at levels well below this $3 \%$ level, so it would generally not be necessary to qualify individual small molecule impurities. It should also be noted that, for impurities that lack the structural elements required for cytotoxicity, it can generally be assumed the safety risk is even lower, so these would be of even less concern than drugcontaining impurities.

ICH M7 provides guidance for assessing risks associated with genotoxic impurities in drug products. However, this guidance is not applicable to drug products that are targeted to cancer patients. While M7 is not applicable, we can look at the risk/benefit principles of this guidance to help in forming the control strategy. One of the key elements of this guideline is that, for drugs that are administered for limited periods of time, higher levels of genotoxic impurities can be tolerated (Table 3). Many oncology drugs are administered over a very short time so patient exposure to any impurity present is very limited. In the example above, the $3 \%$ impurity in the linkerpayload would translate to a patient exposure lower than that outlined in ICH M7 in most cases.

\section{Impurity Control in the DS}

As mentioned above, non-conjugated impurities originating from the production of linker-drug intermediate are typically removed during the purification and isolation of DS. The conjugation of the linker drug to the mAb is the last chemical bond forming step in the ADC process. Most processes-related impurities originating at this step should also be removed during the purification steps. Because these processes related impurities originate at this last bond forming step, they could be present at a higher level than those impurities coming from the linker-drug. Thus, it will normally be more important to demonstrate their control. Conjugation process-related impurities typically include organic solvents, reagents and their by-products, processing aids, and, possibly, degradation products. The purification step's ability to remove these impurities should be demonstrated during development and, much like impurities in other biologic products, would not necessarily need to be addressed in the drug substance specification. One class of non-conjugated impurities that is worth special attention is the linker-drug and its quenched derivatives or byproducts. This is typically encountered in ADC programs due to stoichiometric excess of intermediate used to ensure timely completion of the conjugation reactions. A properly designed UF/DF step should effectively clear these impurities as well, so that they will be well below the ICH Q3A limits and below any level of concern. This can generally be demonstrated during process development. In some situations, additional purification steps such as column chromatography may also be utilized, and these unit operations can provide additional levels of clearance for non-conjugated linker-drug impurities. This type of assessment can also be used to demonstrate that other impurities from the conjugation process, including organic solvents, reagents, reaction by-products, and elemental impurities, are effectively cleared and present little risk.

Figure 3 presents a decision tree that summarizes the approaches discussed above.

\section{Stability of the ADC (as It Relates to Small Molecule Degradants)}

A unique aspect of ADCs that must be considered is the propensity of the DS and DP to release free drug and degradation products that contain the drug. Again, it is the presence of the resulting unconjugated drug that is the highest concern due to the associated risk of toxicity. In

Table 4. Impurity Dose Based on Level of Conjugatable Impurities in the Linker-Drug Intermediate

\begin{tabular}{|c|c|c|c|c|c|c|c|}
\hline \multirow{2}{*}{$\begin{array}{l}\text { Impurity level in } \\
\text { linker-drug }\end{array}$} & \multirow{2}{*}{$\begin{array}{l}\text { Maximum impurity level } \\
\text { in } \mathrm{DS}^{\mathrm{a}}(\mathrm{wt} / \mathrm{wt} \%)\end{array}$} & \multicolumn{2}{|c|}{ ADC $5 \mathrm{mg}$ dose } & \multicolumn{2}{|c|}{ ADC $50 \mathrm{mg}$ dose } & \multicolumn{2}{|c|}{ ADC $500 \mathrm{mg}$ dose } \\
\hline & & $\begin{array}{l}\text { Maximum } \\
\text { impurity level }\end{array}$ & $\begin{array}{l}\text { Maximum daily } \\
\text { impurity level }\end{array}$ & $\begin{array}{l}\text { Maximum } \\
\text { impurity level }\end{array}$ & $\begin{array}{l}\text { Maximum daily } \\
\text { impurity level }\end{array}$ & $\begin{array}{l}\text { Maximum } \\
\text { impurity level }\end{array}$ & $\begin{array}{l}\text { Maximum daily } \\
\text { impurity level }\end{array}$ \\
\hline $3 \%$ & $1.5 \mu \mathrm{g} / \mathrm{mg}$ DS $(0.15 \%)$ & $7.5 \mu \mathrm{g} / \mathrm{dose}$ & $0.36 \mu \mathrm{g} / \mathrm{day}$ & $75 \mu \mathrm{g} / \mathrm{dose}$ & $3.6 \mu \mathrm{g} / \mathrm{day}$ & $0.75 \mathrm{mg} /$ dose & $36.0 \mu \mathrm{g} / \mathrm{day}$ \\
\hline $1 \%$ & $0.5 \mu \mathrm{g} / \mathrm{mg}$ DS $(0.05 \%)$ & $2.5 \mu \mathrm{g} / \mathrm{dose}$ & $0.1 \mu \mathrm{g} / \mathrm{day}$ & $25 \mu \mathrm{g} / \mathrm{dose}$ & $1.2 \mu \mathrm{g} /$ day & $0.25 \mathrm{mg} / \mathrm{dose}$ & $12.0 \mu \mathrm{g} / \mathrm{day}$ \\
\hline $0.5 \%$ & $0.25 \mu \mathrm{g} / \mathrm{mg}$ DS $(0.025 \%)$ & $1.25 \mu \mathrm{g} / \mathrm{dose}$ & $0.06 \mu \mathrm{g} /$ day & $12.5 \mu \mathrm{g} / \mathrm{dose}$ & $0.6 \mu \mathrm{g} /$ day & $0.125 \mathrm{mg} /$ dose & $6.0 \mu \mathrm{g} /$ day \\
\hline $0.1 \%$ & $0.05 \mu \mathrm{g} / \mathrm{mg}$ DS $(0.005 \%)$ & $0.25 \mu \mathrm{g} /$ dose & $0.01 \mu \mathrm{g} / \mathrm{day}$ & $2.5 \mu \mathrm{g} / \mathrm{dose}$ & $0.12 \mu \mathrm{g} /$ day & $0.025 \mathrm{mg} / \mathrm{dose}$ & $1.2 \mu \mathrm{g} / \mathrm{day}$ \\
\hline
\end{tabular}

${ }^{a}$ Calculations were determined by using the Eq. $\mathrm{X}$ 


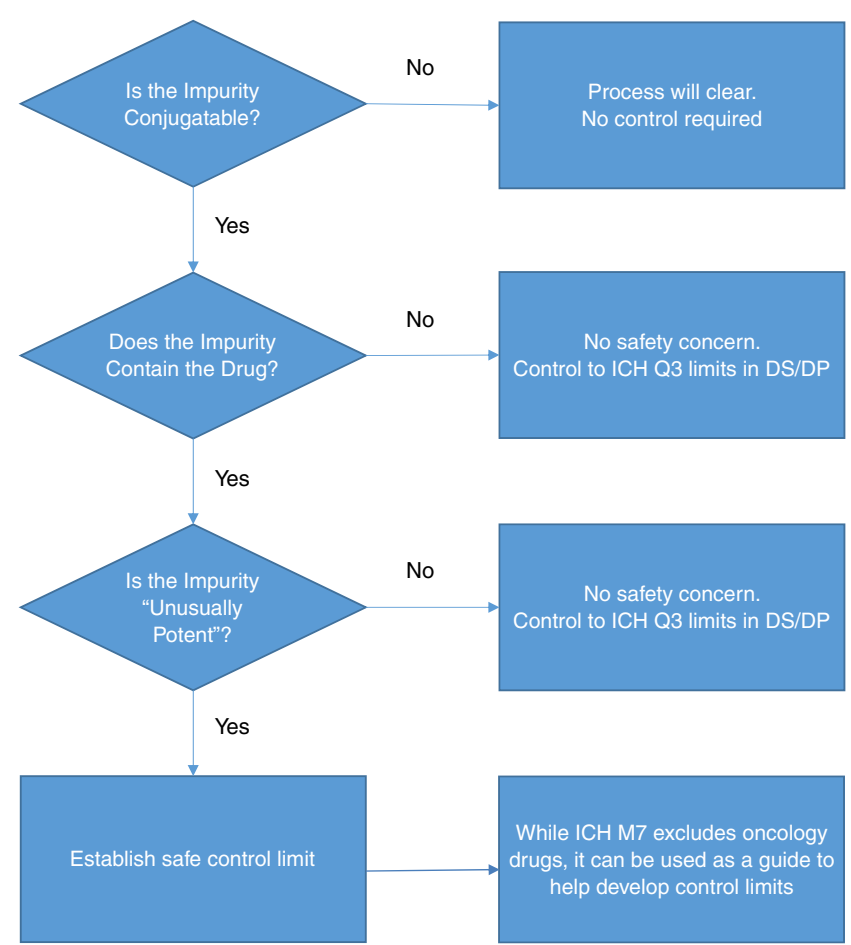

Fig. 3. Decision tree for assessment of impurities in linker-drug intermediates

general, the drug itself is the species with the highest toxicity risk, so treating all drug-related degradants as equivalent to the drug can be viewed as a worst-case assumption.

It is informative to think about the maximum amount of free drug that could be generated if, in the highly improbable but worst case scenario, all the drug fell off the ADC. If this scenario produces an amount of free drug that is below the limits established in $\mathrm{ICH} \mathrm{Q} 3 \mathrm{~A} / \mathrm{B}$, than a more reasonable scenario of partial degradation, then it should be well below this value. The maximum amount possible, expressed as wt/ wt \% , can easily be calculated using Eq. 2, while the absolute quantity of drug can be calculated using Eq. 3. For the prototypical ADC discussed above, with a DAR of 4, drug molecular weight of 1000, and ADC molecular weight of 160,000 , the maximum amount of drug possible corresponds to $2.5 \%$ of the total ADC dose. Based on this analysis, it is clear that an assessment of the stability of the ADC toward degradation should be part of any ADC development program in order to verify control. Depending on the specific ADC, the degradants of concern could be the free drug itself or some chemically modified version.

$\%$ Free Drug $(\max )=\mathrm{DAR} \times \frac{\mathrm{drug} \mathrm{MW}}{\mathrm{ADC} \mathrm{MW}} \times 100$

Free Drug $\left(\max , \frac{\mathrm{mg}}{\text { dose }}\right)=\mathrm{DAR} \times \frac{\text { drug MW }}{\text { ADC MW }} \times \operatorname{Dose}\left(\frac{\mathrm{mg}}{\text { dose }}\right)$

If the ADC dose were $50 \mathrm{mg}$ every 21 days, then the maximum possible level of free drug would be $1.25 \mathrm{mg} / \mathrm{dose}$ or $60 \mu \mathrm{g} /$ day. A more reasonable amount of degradation at the end of shelf life of $10 \%$ loss of drug would result in a daily exposure of only $6 \mu \mathrm{g} / \mathrm{day}$. Table 4 illustrates how the level varies as a function of ADC dose. Applying worst-case scenarios such as this along with an understanding of the toxicity of the drug, one can assess the level of risk that degradation presents. In this example, if doses of $1.25 \mathrm{mg}$ of drug every 3 weeks or $60 \mu \mathrm{g}$ daily of drug were well tolerated in animals, then drug release would not be considered a significant safety concern. On the other hand, the loss of drug is expected to significantly decrease the activity of the ADC, so from the standpoint of product potency, this level of drug loss would be concerning. Regardless of the results of the assessment above, compliance with $\mathrm{ICH}$ Q3A and Q3B would normally remain an expectation.

Table 5 If it is determined that the amount of free drug at the end of the desired shelf-life is excessive, that is, drug loss reduces the potency of the DP, produces impurity levels that present a safety concern, or approaches ICH limits, several options are available. These include the following: (1) reducing the shelf-life to the a period that is short enough so that these concerns are removed; (2) changing the storage conditions; (3) changing the formulation; (4) changing the presentation, e.g., developing a lyophilized product; or (5) adding purification steps, e.g., to remove degradants from DS prior to DP manufacture. If, at the time of licensure, it can be demonstrated that there is little risk of free drug-related impurities, then it may be appropriate to remove tests for free drug-related impurities from the DP release and stability specifications. Figure 4 presents a decision tree that illustrates the thought process for determining how to treat small molecule degradants in ADC DS and DP.

\section{CONCLUSION}

ADCs present challenges that are not typically encountered with small molecule drugs or traditional biologic products. Small molecule impurities, whether conjugated to the protein or not, must be addressed. Due to the low molecular weight of the linker and drug compared to the high molecular weight of the ADC, patient exposure to these small molecule impurities is comparatively low. For these drug products, the small molecule impurities can be assessed using a risk-based approach that is consistent with $\mathrm{ICH}$ Q3A and Q3B guidelines. The control of conjugated small molecule impurities is best achieved at the stage of manufacturing the linker-drug rather than the DS or DP. Following this approach, it can be demonstrated that adopting ICH Q3A limits at the stage of linker-drug intermediates is generally not necessary. Non-conjugated small molecule impurities

Table 5. Examples of Worst Case DP Degradant (Drug) Exposure, Based on Eq. 3

\begin{tabular}{|c|c|c|c|c|c|}
\hline \multicolumn{2}{|c|}{$5 \mathrm{mg}$ dose } & \multicolumn{2}{|c|}{$50 \mathrm{mg}$ dose } & \multicolumn{2}{|c|}{$500 \mathrm{mg}$ dose } \\
\hline $\begin{array}{l}\text { Free } \\
\text { Drug } \\
(\max )\end{array}$ & $\begin{array}{l}\text { Daily drug } \\
\text { exposure } \\
(\max )\end{array}$ & $\begin{array}{l}\text { Free } \\
\text { Drug } \\
(\max )\end{array}$ & $\begin{array}{l}\text { Daily drug } \\
\text { exposure } \\
\text { (max) }\end{array}$ & $\begin{array}{l}\text { Free } \\
\text { Drug } \\
(\max )\end{array}$ & $\begin{array}{l}\text { Daily drug } \\
\text { exposure } \\
(\max )\end{array}$ \\
\hline $125 \mu \mathrm{g}$ & $6 \mu \mathrm{g} /$ day & $1.25 \mathrm{mg}$ & $60 \mu \mathrm{g} /$ day & $12.5 \mathrm{mg}$ & $600 \mu \mathrm{g} / \mathrm{day}$ \\
\hline
\end{tabular}




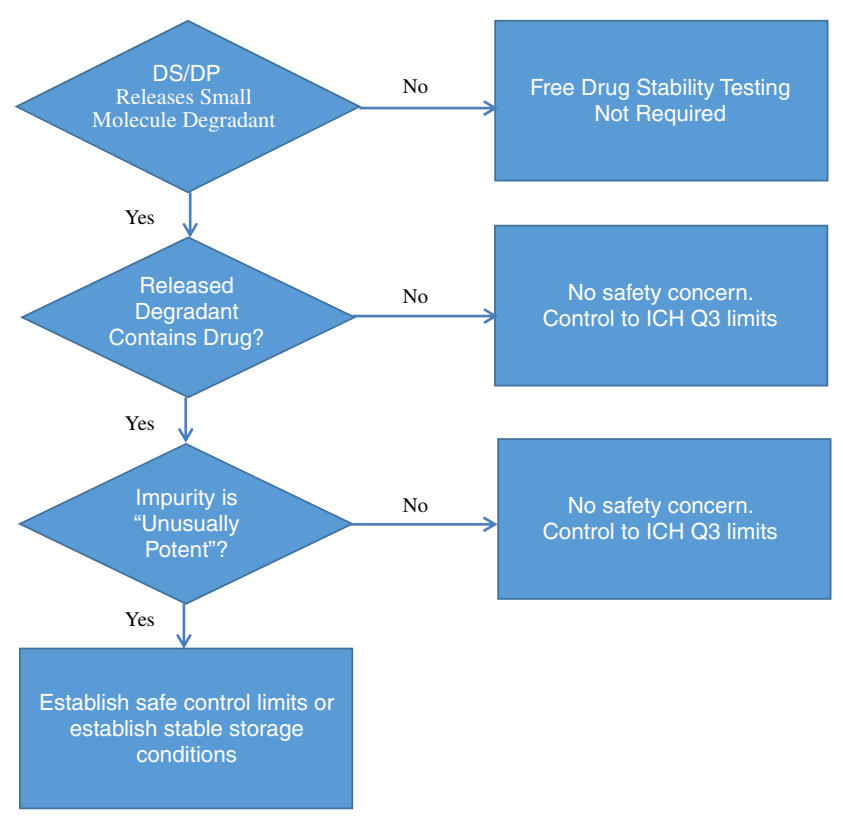

Fig. 4. Decision tree for assessment of small molecule degradants in ADC DS and DP

including free drug are generally cleared effectively by typical manufacturing processes. The purification steps assure compliance with ICH Q3A for non-conjugated impurities. If linker-drug stability after conjugation is demonstrated, then ICH Q3B compliance is assured, and removal of tests for small molecule impurities from release and stability specifications can be justified.

\section{ACKNOWLEDGEMENTS}

The authors would like to thank Andrew Teasdale (AstraZeneca) and Ron Ogilvie (Pfizer) for discussions and edits that greatly improved the quality of this manuscript.

Open Access This article is distributed under the terms of the Creative Commons Attribution 4.0 International License (http://creativecommons.org/licenses/by/4.0/), which permits unrestricted use, distribution, and reproduction in any medium, provided you give appropriate credit to the original author(s) and the source, provide a link to the Creative Commons license, and indicate if changes were made.

\section{REFERENCES}

1. Jackson D. Processes for constructing homogeneous antibody drug conjugates. Org Process Res Dev. 2016;20(5):852-66. https:// doi.org/10.1021/acs.oprd.6b00067.

2. Diamantis N, Banerji U. Antibody-drug conjugates-an emerging class of cancer treatment. Br J Cancer. 2016;114(4):362-7. https:// doi.org/10.1038/bjc.2015.435.

3. ADC Review, Journal of antibody-drug conjugates, (ISSN 2327-0152).

4. Antibody-drug conjugates: fundamentals, drug development, and clinical outcomes to target cancer, Kenneth J. Olivier, Sara A. Hurvitz, Editors: John Wiley \& Sons, Inc. p. 1-560.

5. Kurnik RT, AW Y, Blank GS, Burton AR, Smith D, Athalye AM, et al. Buffer exchange using size exclusion chromatography, countercurrent dialysis, and tangential flow filtration: models, development, and industrial application. Biotechnol Bioeng. 1995;45(2):149-57. https://doi.org/10.1002/bit.260450209. 